\title{
Response errors explain the failure of independent-channels models of perception of temporal order
}

\author{
Miguel A. García-Pérez * and Rocío Alcalá-Quintana \\ Departamento de Metodología, Facultad de Psicología, Universidad Complutense, Madrid, Spain
}

\section{Edited by:}

Peter J. Bex, Harvard University, USA

Reviewed by:

Luis Lesmes, Salk Institute, USA

Edward Vul, Massachusetts Institute

of Technology, USA

*Correspondence:

Miguel A. García-Pérez, Departamento de Metodología,

Facultad de Psicología, Universidad

Complutense, Campus de

Somosaguas, 28223 Madrid, Spain.

e-mail:miguel@psi.ucm.es
Independent-channels models of perception of temporal order (also referred to as threshold models or perceptual latency models) have been ruled out because two formal properties of these models (monotonicity and parallelism) are not borne out by data from ternary tasks in which observers must judge whether stimulus $A$ was presented before, after, or simultaneously with stimulus B. These models generally assume that observed responses are authentic indicators of unobservable judgments, but blinks, lapses of attention, or errors in pressing the response keys (maybe, but not only, motivated by time pressure when reaction times are being recorded) may make observers misreport their judgments or simply guess a response. We present an extension of independent-channels models that considers response errors and we show that the model produces psychometric functions that do not satisfy monotonicity and parallelism. The model is illustrated by fitting it to data from a published study in which the ternary task was used. The fitted functions describe very accurately the absence of monotonicity and parallelism shown by the data. These characteristics of empirical data are thus consistent with independent-channels models when response errors are taken into consideration. The implications of these results for the analysis and interpretation of temporal order judgment data are discussed.

Keywords: temporal order judgment, simultaneity judgment, response errors, audiovisual events, experimental methods, model identifiability

\section{INTRODUCTION}

Judgments of the temporal order or simultaneity of two stimuli are collected in studies of prior entry, multisensory integration, or causality perception (Schneider and Bavelier, 2003; Stetson et al., 2006; Spence and Parise, 2010; Vroomen and Keetels, 2010; Yates and Nicholls, 2011) and, more generally, in research on perception of temporal order (Sternberg and Knoll, 1973; Occelli et al., 2011). The two stimuli that are involved may pertain to different sensory modalities but, without loss of generality, we will assume the audiovisual case in the following description. In these experiments, visual and auditory stimuli are presented with a range of auditory delays (or stimulus onset asynchrony, SOA), defined as the difference between the onset of the auditory stimulus and that of the visual stimulus. In the ternary version of the simultaneity judgment task (SJ3 task; Ulrich, 1987), the observer must judge whether the auditory stimulus was presented before, after, or simultaneously with the visual stimulus, yielding "audio-first" (AF), "video-first" (VF), or "simultaneous" (S) responses.

Models of timing judgments fall within the class of independent-channels models described by Sternberg and Knoll (1973), in which signals from the two stimuli reach a central mechanism with randomly distributed arrival latencies. At the central mechanism, the judgment arises on application of a decision rule to the arrival-time difference between the signals. Sternberg and Knoll derived general properties of independent-channels models without making assumptions about the distribution of arrival latencies or about the form of the decision rule. With explicit assumptions about these components, independent-channels models yield expressions for the psychometric functions $\Psi_{\mathrm{AF}}, \Psi_{\mathrm{S}}$, and $\Psi_{\mathrm{VF}}$, respectively describing how the probabilities of AF, S, and VF judgments vary with auditory delay. Sternberg and Knoll also showed that the attention-switching models of Kristofferson and Allan (1973) can be cast in terms of independent-channels models and, thus, they do not represent an essentially different type of models as regards the issues addressed in this paper.

A theoretically important feature of independent-channels models of perception of temporal order is that they entail a representation of the processes governing observed performance at timing judgment tasks. Model parameters are thus straightforwardly interpretable as reflecting characteristics of the distributions of arrival latencies (determined by sensory transmission and perceptual processing), and they also characterize the observer as a decision maker whose judgments rely on sensory information, subject to limitations imposed by the temporal resolution with which observers can tell small differences in arrival latency. These models are thus useful in studies of prior entry or temporal recalibration because parameter estimates directly indicate how experimental manipulations affect either the distributions of arrival latencies or the operating temporal resolution of the observer.

However, empirical tests of the models revealed their inadequacy because data failed to satisfy certain formal properties that should manifest in SJ3 tasks. For instance, Allan (1975) showed 
that independent-channels models imply that $\Psi_{\mathrm{VF}}$ should differ from $1-\Psi_{\mathrm{AF}}$ only by horizontal translation (a property called parallelism), but her data did not bear out this prediction. Ulrich (1987) showed that these models (as well as attention-switching models) also imply that $\Psi_{\mathrm{VF}}$ should be a strictly increasing function whereas $\Psi_{\mathrm{AF}}$ should be a strictly decreasing function (a property called monotonicity), he derived further properties of the models, and he also reported data violating them. Alternative models were proposed by Allan and by Ulrich and yet another model was later proposed by Jaskowski (1991), but these alternative models are not without problems either. For instance, Allan's model is an amended attention-switching model that includes guessing mechanisms and predicts four-limbed linear psychometric functions always including a zero-slope limb; the model can account for lack of parallelism but not for lack of monotonicity, but piecewise linear psychometric functions including a flat portion for $\mathrm{AF}$ and VF data have never been reported. Ulrich only sketched an amended attention-switching model that he regarded as "very promising" but, to the authors' knowledge, development and test of this model was never published. Finally, Jaskowski's model is an amended independent-channels model with dual streams on the assumption that judgments of successiveness and judgments of temporal order arise from independent processing paths, an assumption whose validity had been empirically disproved by Allan (1975). Perhaps because of these shortcomings, none of these alternative models has been subsequently used to fit SJ3 data (for the single exception that we are aware of, see Jaskowski, 1993).

By putting aside independent-channels models and their variants for the analysis of timing judgment data, benefits of the interpretability of their parameters are lost. But data must nevertheless be analyzed somehow and current practice consists of fitting arbitrary functions of adequate shape to the data. Thus, survival Gaussians are typically fitted to accommodate the decreasing trend of AF data (or equivalent data when other sensory modalities are involved), cumulative logistic or Gaussian functions are used to fit the increasing trend of VF data (or their equivalent for other sensory modalities), and Gaussians or piecewise cumulativesurvival Gaussians are fitted to inverted-U shaped S data (Shore et al., 2002; Stone et al., 2001; Spence et al., 2003; Harrar and Harris, 2008; van Eijk et al., 2008, 2010; Vatakis et al., 2008; Fujisaki and Nishida, 2009; Yates and Nicholls, 2009, 2011; Nicholls et al., 2011). Although separate functions fitted in this way can capture lack of parallelism of AF and VF data, the fitted functions are monotonic and cannot accommodate lack of monotonicity in the data. Also, and for lack of a theoretical framework within which these arbitrary functions are derived, their parameters only describe the data themselves with no links to interpretable parameters that might indicate the characteristics of underlying processes. Fitting to data models with interpretable parameters would thus be very useful.

One aspect that has never been considered theoretically in any depth is that observers occasionally have lapses of attention (yielding pure guesses as responses) or make errors upon pressing the response keys (sometimes yielding unexpected responses). Besides the observers' spontaneous reports of these events at the end of the experimental session, inspection of raw data typically shows evidence of them, namely, unexpected AF responses at long positive auditory delays (where VF responses should always occur) and unexpected VF responses at long negative auditory delays (where AF responses should always occur). The arbitrary functions referred to above are also typically fitted without allowance for asymptote parameters that account for these events (for rare exceptions, see Spence et al., 2003; van Eijk et al., 2008, 2010) and Allan's and Ulrich's analyses of the properties of independent-channels models did not consider these events either. This contrasts with the tradition in other fields (e.g., visual psychophysics), where the importance of including lapse or finger-error parameters in psychometric functions is acknowledged (see Swanson and Birch, 1992; Wichmann and Hill, 2001).

In this paper we explore whether an extension of independentchannels models that considers these factors can account for data showing lack of monotonicity and lack of parallelism, which would generally be taken as ruling out such models entirely. Consideration of parameters to represent these factors in the ternary SJ3 task is slightly more complex than in the binary tasks involved in visual psychophysics. Thus, we first present an amended independentchannels model that includes parameters to represent response errors in a realistic manner and we show that parallelism and monotonicity are no longer properties of the model when response errors are considered. Subsequently, we show that the model fits SJ3 data adequately and, hence, that empirically observed deviations from these properties can be explained as a consequence of these events. The importance of this result lies in that independentchannels models can be reinstated and, thus, temporal order judgment data can be analyzed in terms of the interpretable parameters of these models. Thus, data can be used to make direct inferences about the underlying processes (e.g., distributions of arrival latencies across experimental conditions in studies of prior entry) instead of merely used to obtain quantitative indices that describe empirical performance through arbitrary functions that lack theoretical underpinnings.

\section{THE MODEL}

Our starting point is an independent-channels model similar to a perceptual latency model with a threshold decision process (Allan, 1975) or to a triggered-moment model (Schneider and Bavelier, 2003), with some modifications. The arrival latencies $T_{\mathrm{v}}$ and $T_{\mathrm{a}}$ of visual and auditory signals are random variables with densities $g_{\mathrm{v}}$ and $g_{\mathrm{a}}$, respectively. In contrast to the Gaussian assumption in the models just mentioned, we will assume shifted exponential distributions given by

$g_{i}(t)=\lambda_{i} \exp \left[-\lambda_{i}\left(t-\left(\Delta t_{i}+\tau_{i}\right)\right)\right], t \geqslant \Delta t_{i}+\tau_{i}, \quad i \in\{\mathrm{v}, \mathrm{a}\}$,

where $\Delta t_{i}$ (in ms) is the actual onset of the corresponding signal, $\lambda_{i}$ (in $\mathrm{ms}^{-1}$ ) is the rate parameter of the distribution (indicating how fast probability density decreases as $t$ increases), and $\tau_{i}$ (in $\mathrm{ms}$ ) is a further processing delay. Exponential distributions are commonly assumed to describe arrival latencies and peripheral processing times (Heath, 1984; Colonius and Diederich, 2011; Diederich and Colonius, 2011), whose mean is thus $1 / \lambda_{i}+\tau_{i}$ and whose $\mathrm{SD}$ is $1 / \lambda_{i}$.

Without loss of generality, we set the origin of time at the onset of the visual stimulus so that $\Delta t_{\mathrm{v}}=0$ and, thus, $\Delta t \equiv \Delta t_{\mathrm{a}}$ 
is the auditory delay manipulated experimentally, where $\Delta t<0$ $(\Delta t>0)$ reflects that the auditory signal precedes (follows) the visual signal. Figure 1A shows sample distributions for $\lambda_{\mathrm{a}}=1 / 60$, $\lambda_{\mathrm{v}}=1 / 30, \tau_{\mathrm{a}}=20$, and $\tau_{\mathrm{v}}=40$ when the auditory delay is $\Delta t=50$. On a given trial, arrival latencies are realizations of these distributions and the observer's judgment arises from a decision rule applied to the arrival-time difference $D=T_{\mathrm{a}}-T_{\mathrm{v}}$, which has a bilateral exponential distribution given by

$f(d ; \Delta t)=\left\{\begin{array}{ll}\frac{\lambda_{\mathrm{a}} \lambda_{\mathrm{v}}}{\lambda_{\mathrm{a}}+\lambda_{\mathrm{v}}} \exp \left[\lambda_{\mathrm{v}}(d-\Delta t-\tau)\right] & \text { if } d \leqslant \Delta t+\tau \\ \frac{\lambda_{\mathrm{a}} \lambda_{\mathrm{v}}}{\lambda_{\mathrm{a}}+\lambda_{\mathrm{v}}} \exp \left[-\lambda_{\mathrm{a}}(d-\Delta t-\tau)\right] & \text { if } d>\Delta t+\tau\end{array}\right.$,

where $\tau=\tau_{\mathrm{a}}-\tau_{\mathrm{v}}$. Figure 1B shows the distribution of arrivaltime differences for the case in Figure 1A. A resolution parameter $\delta$ - which was referred to as threshold by Sternberg and Knoll (1973), Allan (1975), and Ulrich (1987) and as moment duration by Schneider and Bavelier (2003) - limits discriminability so that an AF judgment occurs when $D$ is sufficiently large and negative $(D<-\delta)$, a VF judgment occurs when $D$ is sufficiently large and positive $(D>\delta)$, and an $S$ judgment occurs when the arrival-time difference is below the resolution limit $(-\delta \leq D \leq \delta)$.

For the example in Figure $1 \mathrm{~B}$, where $\delta=100$, the probability of AF, S, and VF judgments are, respectively, 0.0044, 0.7880, and 0.2076 (as indicated in Figure 1B; see also the circles on the curves of Figure 1C). These probabilities change with auditory delay $\Delta t$ and Figure 1C shows complete psychometric functions. To obtain them, first note that the cumulative distribution for $D$ is

$$
\begin{aligned}
F(d ; \Delta t) & =\int_{-\infty}^{d} f(z ; \Delta t) \mathrm{d} z \\
& =\left\{\begin{array}{ll}
\frac{\lambda_{\mathrm{a}}}{\lambda_{\mathrm{a}}+\lambda_{\mathrm{v}}} \exp \left[\lambda_{\mathrm{v}}(d-\Delta t-\tau)\right] & \text { if } d \leqslant \Delta t+\tau \\
1-\frac{\lambda_{\mathrm{v}}}{\lambda_{\mathrm{a}}+\lambda_{\mathrm{v}}} \exp \left[-\lambda_{\mathrm{a}}(d-\Delta t-\tau)\right] & \text { if } d>\Delta t+\tau
\end{array},\right.
\end{aligned}
$$

where $f$ is given by Eq. 2. Then,

$$
\begin{aligned}
\Psi_{\mathrm{AF}}(\Delta t) & =\int_{-\infty}^{-\delta} f(z ; \Delta t) \mathrm{d} z=F(-\delta ; \Delta t) \\
\Psi_{\mathrm{S}}(\Delta t) & =\int_{-\delta}^{\delta} f(z ; \Delta t) \mathrm{d} z=F(\delta ; \Delta t)-F(-\delta ; \Delta t) \\
\Psi_{\mathrm{VF}}(\Delta t) & =\int_{\delta}^{\infty} f(z ; \Delta t) \mathrm{d} z=1-F(\delta ; \Delta t) .
\end{aligned}
$$

Clearly, $\Psi_{\mathrm{VF}}$ and $\Psi_{\mathrm{AF}}$ are both monotonic and parallelism holds because $\Psi_{\mathrm{VF}}(\Delta t)=1-\Psi_{\mathrm{AF}}(\Delta t+2 \delta)$, that is, the blue curve in Figure 1C differs from an upside-down reversal of the red curve only by a lateral shift.

Equations $4 \mathrm{a}-4 \mathrm{c}$ give the probabilities of the unobservable AF, $\mathrm{S}$, and VF judgments as a function of auditory delay, but the probabilities of observed AF, S, and VF responses may differ from them. For instance, errors in pressing the response keys make the observer's response differ from the judgment that was made. In addition, blinks or lapses of attention preclude judgments at all

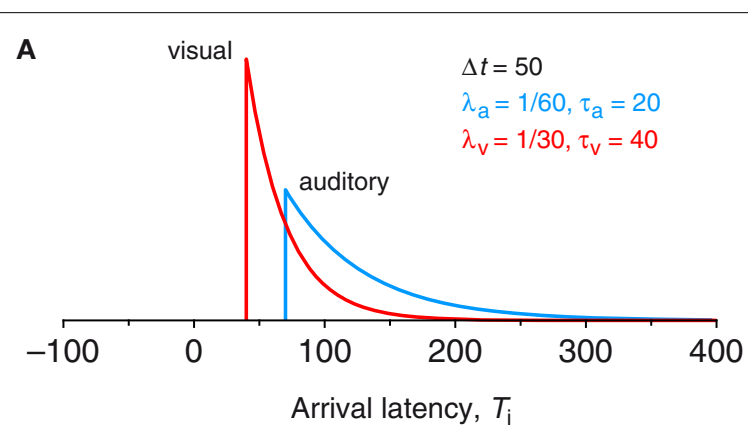

B

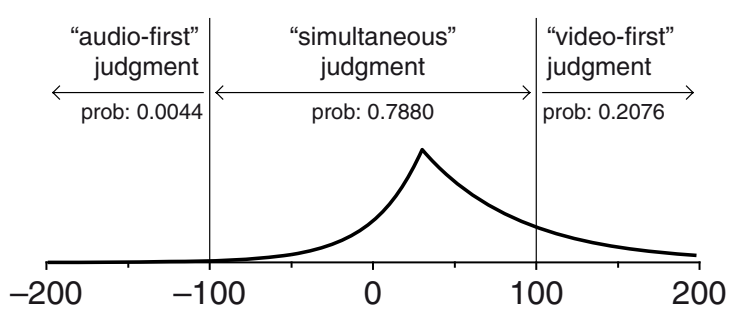

Arrival-time difference, $D=T_{\mathrm{a}}-T_{\mathrm{v}}$

C

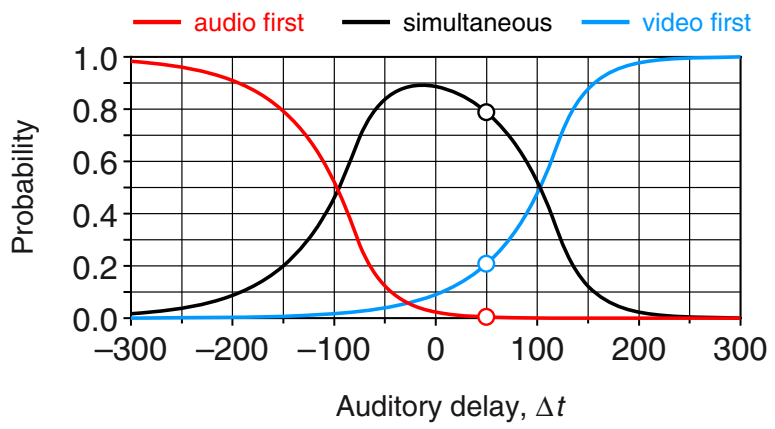

D

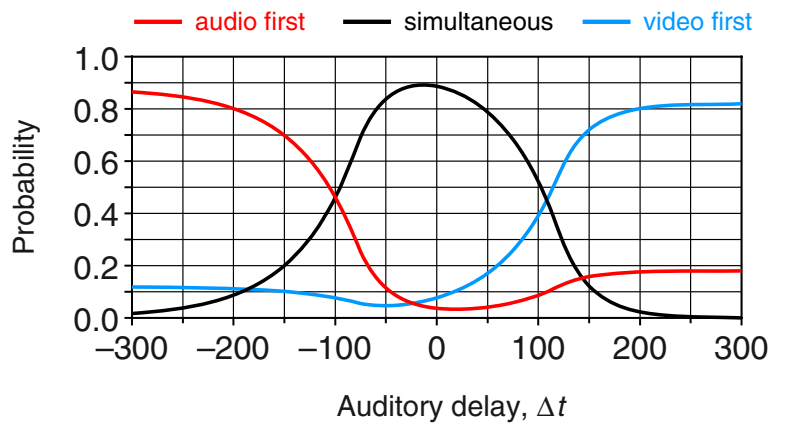

FIGURE 1 | Model of timing judgments. (A) Exponential distributions for the arrival latency of a visual stimulus (red curve) presented at time 0 and an auditory stimulus (blue curve) presented at time $\Delta t=50 \mathrm{~ms}$. Parameters as indicated. (B) Bilateral exponential distribution of arrival-time differences and boundaries on the decision space (vertical lines, at $D= \pm \delta$ with $\delta=100$ ), determining the probability of each type of judgment. (C). Error-free psychometric functions for each type of response as a function of auditory delay. Circles denote the probabilities indicated in (B) for $\Delta t=50 \mathrm{~ms}$. (D) Psychometric functions when response errors occur as described in the text. 
and force the observer to guess a response that may not match the judgment that would have been made in the absence of a lapse. We will refer to these misreports as response errors and lapses, respectively, and we will first describe how to incorporate the former into the model.

Let $\varepsilon_{\mathrm{AF}}, \varepsilon_{\mathrm{S}}$, and $\varepsilon_{\mathrm{VF}}$ be the probabilities (or error rates) that the observer misreports AF, S, and VF judgments, respectively, as a result of an error in pressing the response keys. These errors may actually differ across the judgments that were made, if only because the layout of the response interface may make the observers more likely to misreport one of the judgments and, in such cases, also more likely to misreport it in a particular form. Since misreporting any given judgment can take two forms (i.e., there are two possible intruding responses), let $\kappa_{\mathrm{A}-\mathrm{B}}$ be the conditional probability of misreporting an $\mathrm{A}$ judgment as a $\mathrm{B}$ response so that $\kappa_{\mathrm{A}-\mathrm{C}}=1-\kappa_{\mathrm{A}-\mathrm{B}}$ is the conditional probability of misreporting an A judgment as a $\mathrm{C}$ response. Only three conditional probabilities are free parameters, say, $\kappa_{S-A F}, \kappa_{V F-A F}$, and $\kappa_{A F-S}$, because $\kappa_{\mathrm{S}-\mathrm{VF}}=1-\kappa_{\mathrm{S}-\mathrm{AF}}, \kappa_{\mathrm{VF}-\mathrm{S}}=1-\kappa_{\mathrm{VF}-\mathrm{AF}}$, and $\kappa_{\mathrm{AF}-\mathrm{VF}}=1-\kappa_{\mathrm{AF}-\mathrm{S}}$. The model incorporating response errors thus becomes

$$
\begin{aligned}
\Psi_{\mathrm{AF}}^{*}(\Delta t)= & \left(1-\varepsilon_{\mathrm{AF}}\right) \Psi_{\mathrm{AF}}(\Delta t)+\varepsilon_{\mathrm{S}} \kappa_{\mathrm{S}-\mathrm{AF}} \Psi_{\mathrm{S}}(\Delta t) \\
& +\varepsilon_{\mathrm{VF}} \kappa_{\mathrm{VF}-\mathrm{AF}} \Psi_{\mathrm{VF}}(\Delta t) \\
\Psi_{\mathrm{S}}^{*}(\Delta t)= & \varepsilon_{\mathrm{AF}} \kappa_{\mathrm{AF}-\mathrm{S}} \Psi_{\mathrm{AF}}(\Delta t)+\left(1-\varepsilon_{\mathrm{S}}\right) \Psi_{\mathrm{S}}(\Delta t) \\
& +\varepsilon_{\mathrm{VF}}\left(1-\kappa_{\mathrm{VF}-\mathrm{AF}}\right) \Psi_{\mathrm{VF}}(\Delta t) \\
\Psi_{\mathrm{VF}}^{*}(\Delta t)= & \varepsilon_{\mathrm{AF}}\left(1-\kappa_{\mathrm{AF}-\mathrm{S}}\right) \Psi_{\mathrm{AF}}(\Delta t)+\varepsilon_{\mathrm{S}}\left(1-\kappa_{\mathrm{S}-\mathrm{AF}}\right) \Psi_{\mathrm{S}}(\Delta t) \\
& +\left(1-\varepsilon_{\mathrm{VF}}\right) \Psi_{\mathrm{VF}}(\Delta t),
\end{aligned}
$$

where $\Psi_{\mathrm{AF}}, \Psi_{\mathrm{S}}$, and $\Psi_{\mathrm{VF}}$ on the right-hand sides are given by Eqs 4a-4c. Figure 1D shows the resultant psychometric functions for an observer with a relatively high error rate whereby $\mathrm{VF}$ judgments are misreported as AF responses $\left(\varepsilon_{\mathrm{VF}}=0.18\right.$ and $\kappa_{\mathrm{VF}-\mathrm{AF}}=1$ ), a weaker error rate whereby $\mathrm{AF}$ judgments are misreported as VF responses $\left(\varepsilon_{\mathrm{AF}}=0.12\right.$ and $\left.\kappa_{\mathrm{AF}-\mathrm{S}}=0\right)$, and no misreports of $S$ judgments $\left(\varepsilon_{S}=0\right)$. Note that the blue and red curves in Figure 1D are non-monotonic and do not satisfy parallelism (after upside-down reversal). Note also that an absence of errors (i.e., $\varepsilon_{\mathrm{AF}}=\varepsilon_{\mathrm{S}}=\varepsilon_{\mathrm{VF}}=0$ ) renders the model in Eqs $4 \mathrm{a}-4 \mathrm{c}$, in which responses faithfully indicate judgments.

Equations $4 \mathrm{a}-4 \mathrm{c}$ give the probability of (unobservable) judgments, whereas Eqs $5 a-5 c$ give the probabilities of observed responses. Thus, observed responses potentially reflect mixtures of "authentic" judgments and intrusions due to errors upon reporting judgments of other types. We will refer to the weights of the intruding responses in these mixtures as intrusion parameters. For the example in Figure 1D, Eqs 5a-5c become

$$
\begin{aligned}
\Psi_{\mathrm{AF}}^{*}(\Delta t)= & 0.88 \times \Psi_{\mathrm{AF}}(\Delta t)+0.00 \times \Psi_{\mathrm{S}}(\Delta t) \\
& +0.18 \times \Psi_{\mathrm{VF}}(\Delta t) \\
\Psi_{\mathrm{S}}^{*}(\Delta t)= & 0.00 \times \Psi_{\mathrm{AF}}(\Delta t)+1.00 \times \Psi_{\mathrm{S}}(\Delta t) \\
& +0.00 \times \Psi_{\mathrm{VF}}(\Delta t) \\
\Psi_{\mathrm{VF}}^{*}(\Delta t)= & 0.12 \times \Psi_{\mathrm{AF}}(\Delta t)+0.00 \times \Psi_{\mathrm{S}}(\Delta t) \\
& +0.82 \times \Psi_{\mathrm{VF}}(\Delta t)
\end{aligned}
$$

so that $\Psi_{S}^{*}=\Psi_{\mathrm{S}}$ for lack of intrusions from AF and VF judgments and also for lack of intrusion of $\mathrm{S}$ judgments into AF and $\mathrm{VF}$ responses. In contrast, $\Psi_{\mathrm{AF}}^{*}$ misses $12 \%$ of the authentic AF judgments (which intrude into VF responses) and $\Psi_{\mathrm{VF}}^{*}$ misses $18 \%$ of the authentic VF judgments (which intrude into AF responses).

Our next step to model development considers lapses of attention. These lapses (or blinks, yawns, sneezes, ...) obviously occur randomly across trials and independently of the auditory delay at the trial and also independently of the judgment that would have been made in the absence of a lapse. There is a (conceivably small) probability $\gamma$ that a lapse occurs on some trial and, in such case, an observer can only arbitrarily give AF, $\mathrm{S}$, or $\mathrm{VF}$ responses perhaps with some bias. Let $\beta_{\mathrm{AF}}, \beta_{\mathrm{S}}$, and $\beta_{\mathrm{VF}}=1-\beta_{\mathrm{AF}}-\beta_{\mathrm{S}}$ be the probabilities of these responses in case of a lapse, where $\beta_{\mathrm{AF}}=\beta_{\mathrm{S}}=\beta_{\mathrm{VF}}=1 / 3$ for an unbiased observer (although unbiased guessing behavior cannot be generally assumed in advance). The model incorporating only lapses and the ensuing (potentially biased) guesses thus becomes

$$
\begin{aligned}
\Psi_{\mathrm{AF}}^{+}(\Delta t) & =\gamma \beta_{\mathrm{AF}}+(1-\gamma) \Psi_{\mathrm{AF}}(\Delta t) \\
\Psi_{\mathrm{S}}^{+}(\Delta t) & =\gamma \beta_{\mathrm{S}}+(1-\gamma) \Psi_{\mathrm{S}}(\Delta t) \\
\Psi_{\mathrm{VF}}^{+}(\Delta t) & =\gamma \beta_{\mathrm{VF}}+(1-\gamma) \Psi_{\mathrm{VF}}(\Delta t) .
\end{aligned}
$$

The effect of lapses on the shape of observable psychometric functions can be described without graphical illustration: compared to the psychometric functions in Figure 1C, multiplication by $1-\gamma$ shrinks the range of the functions (i.e., shifts the upper asymptotes of $\Psi_{\mathrm{AF}}$ and $\Psi_{\mathrm{VF}}$ down and also scales $\Psi_{\mathrm{S}}$ down, without affecting the lower asymptotes of any of them) whereas the additive term pushes the functions upwards by a small amount, thus shifting the lower asymptotes upwards.

Now, combining the effects of lapses and response errors into an integrated model is as simple as replacing the unmarked psychometric functions on the right-hand sides of Eqs $7 a-7 c$ with the right-hand sides of Eqs 5a-5c, yielding

$$
\begin{aligned}
\Psi_{\mathrm{AF}}^{*+}(\Delta t)= & \gamma \beta_{\mathrm{AF}}+(1-\gamma)\left[\left(1-\varepsilon_{\mathrm{AF}}\right) \Psi_{\mathrm{AF}}(\Delta t)\right. \\
& \left.+\varepsilon_{S} \kappa_{\mathrm{S}-\mathrm{AF}} \Psi_{\mathrm{S}}(\Delta t)+\varepsilon_{\mathrm{VF}} \kappa_{\mathrm{VF}-\mathrm{AF}} \Psi_{\mathrm{VF}}(\Delta t)\right] \\
\Psi_{\mathrm{S}}^{*+}(\Delta t)= & \gamma \beta_{\mathrm{S}}+(1-\gamma)\left[\varepsilon_{\mathrm{AF}} \kappa_{\mathrm{AF}-\mathrm{S}} \Psi_{\mathrm{AF}}(\Delta t)\right. \\
& \left.+\left(1-\varepsilon_{\mathrm{S}}\right) \Psi_{\mathrm{S}}(\Delta t)+\varepsilon_{\mathrm{VF}}\left(1-\kappa_{\mathrm{VF}-\mathrm{AF}}\right) \Psi_{\mathrm{VF}}(\Delta t)\right] \\
\Psi_{\mathrm{VF}}^{*+}(\Delta t)= & \gamma \beta_{\mathrm{VF}}+(1-\gamma)\left[\varepsilon_{\mathrm{AF}}\left(1-\kappa_{\mathrm{AF}-\mathrm{S}}\right) \Psi_{\mathrm{AF}}(\Delta t)\right. \\
& \left.+\varepsilon_{\mathrm{S}}\left(1-\kappa_{\mathrm{S}-\mathrm{AF}}\right) \Psi_{\mathrm{S}}(\Delta t)+\left(1-\varepsilon_{\mathrm{VF}}\right) \Psi_{\mathrm{VF}}(\Delta t)\right] .
\end{aligned}
$$

The effect of lapses in this integrated model is again analogous to that described above, except that the shrunken range and vertical shift occur for the psychometric functions in Figure 1D instead of those in Figure 1C. There are three reasons why we will not consider this integrated model, all of which pertain to the limited utility of modeling lapses. The first one is that the model for lapses in Eqs 7a-7c violates parallelism but not monotonicity: all that is implied when only lapses occur is vertical shrinkage and vertical shift of the psychometric functions and, thus, lapses cannot possibly account for the non-monotonicity observed in some 
data sets. Second, the integrated model in Eqs $8 \mathrm{a}-8 \mathrm{c}$ is unidentifiable, as lapses and response errors both affect the asymptotes of the psychometric functions in an inextricable way for lack of independent evidence as to how much of the effect found in empirical data is caused by lapses and how much is caused by response errors. Finally, many experiments are designed so as to eliminate the influence of lapses by enabling an additional response key for observers to abort the trial if they missed the stimuli and could not make a judgment. This key is usually programmed so that the trial is placed back in the stack of pending trials for administration at a later time (generally not immediately afterward), and observers are instructed to refrain from using this key if they only were uncertain and wanted to have a second chance. If the commendable precaution to enable such "abort" key is taken, lapses do not need to be modeled at all.

Our decision to use only the model with response errors should not be misconstrued for a denial of the existence of lapses. In the context of our goals in this paper, the potential effects of lapses are absorbed by the error parameters in the model that we will use, and the only consequence is that the estimated values for these parameters cannot be literally interpreted as representing only the probabilities of response errors. This is not a crucial problem, because the relevant parameters in research on prior entry or perception of temporal order are only $\lambda_{\mathrm{a}}, \lambda_{\mathrm{v}}, \tau$, and $\delta$; parameters describing errors or lapses are rarely of any theoretical interest and they are included in the model only to improve the accuracy with which the relevant parameters are estimated.

\section{FITTING THE MODEL TO DATA}

Model parameters were estimated for data reported by van Eijk et al. (2008) from an audiovisual SJ3 task carried out by 11 observers. Only results for data from their flash-click stimulus will be presented here, as data from the other stimulus yielded similar outcomes. The visual component of the stimulus was a white disk flashed for $12 \mathrm{~ms}$ against a dark background and the auditory component was a 12-ms white-noise burst. Auditory delays ranged from -350 to $350 \mathrm{~ms}$ in steps of $50 \mathrm{~ms}$, and 60 trials were administered at each auditory delay.

Model parameters for each observer were sought by maximizing the likelihood equation

$$
L(\boldsymbol{R} \mid \boldsymbol{\theta})=\prod_{i=1}^{N}\left[\Psi_{\mathrm{AF}}^{*}\left(\Delta t_{i}\right)\right]^{A_{i}}\left[\Psi_{\mathrm{S}}^{*}\left(\Delta t_{i}\right)\right]^{\mathrm{S}_{i}}\left[\Psi_{\mathrm{VF}}^{*}\left(\Delta t_{i}\right)\right]^{V_{i}},
$$

where $\boldsymbol{R}$ is the set of empirical responses, $\theta=\left(\lambda_{\mathrm{a}}, \lambda_{\mathrm{V}}, \tau, \delta\right.$, $\left.\varepsilon_{\mathrm{AF}}, \varepsilon_{\mathrm{S}}, \varepsilon_{\mathrm{VF}}, \kappa_{\mathrm{S}-\mathrm{AF}}, \kappa_{\mathrm{VF}-\mathrm{AF}}, \kappa_{\mathrm{AF}-\mathrm{S}}\right)$ is the vector of free parameters, $\left\{\Delta t_{1}, \Delta t_{2}, \ldots, \Delta t_{N}\right\}$ is the set of $N=15$ auditory delays at which responses were collected, and $A_{i}, S_{i}$, and $V_{i}$ are the observed counts of AF, $\mathrm{S}$, and VF responses at $\Delta t_{i}$. Equation 9 was maximized using NAG subroutine E04JYF (Numerical Algorithms Group, 1999), which implements a quasi-Newton algorithm for constrained optimization. The parameter space spanned the ranges $[1 / 200,1]$ for $\lambda_{\mathrm{a}}$ and $\lambda_{\mathrm{V}}$, the range $[-150,150]$ for $\tau$, the range $[0,300]$ for $\delta$, the range $[0,0.8]$ for $\varepsilon_{\mathrm{AF}}, \varepsilon_{S}$, and $\varepsilon_{\mathrm{VF}}$, and the range $[0,1]$ for $\kappa_{\mathrm{AF}-\mathrm{S}}, \kappa_{\mathrm{S}-\mathrm{AF}}$, and $\kappa_{\mathrm{VF}-\mathrm{AF}}$. Two or three initial values were defined for each parameter, which were evenly spaced within the search space for that parameter. Initial values for each parameter were factorially combined to yield $3^{4} \times 2^{6}$ starting points in the 10 -dimensional parameter space and the maximization routine ran for each of these starting points, yielding in each case a vector of estimates and a divergence index. On completion, we took the vector of estimates for which divergence was lowest and the likelihood-ratio statistic $G^{2}$ was computed as a measure of goodness-of-fit because this statistic is the one that maximum-likelihood estimates minimize (Collett, 2003, pp. 87-88). Thus, we estimated parameters and measured the goodness of the fit using the same "currency" (Wichmann and Hill, 2001). The asymptotic distribution of all goodness-of-fit statistics is known to yield inaccurate significance levels when expected frequencies are small (García-Pérez, 1994; García-Pérez and NúñezAntón, 2001, 2004) and this is a common encounter when fitting psychometric functions. For this reason, significance levels were obtained through parametric bootstrap by simulating 5000 data sets using the estimated parameters for each observer and the same number of auditory delays and trials per delay as in the actual experiment.

Figure 2 shows empirical data and fitted functions for each observer, and also shows a summary panel for average data and average fitted curves for all observers (which we include only because this is the format in which alternative fitted curves were reported by van Eijk et al., 2008). Table 1 lists parameter estimates as well as the value and $p$-value of the $G^{2}$ statistic for each observer. Perhaps the most salient aspect of Figure $\mathbf{2}$ is that model curves follow the path of the data accurately, accommodating systematic deviations from monotonicity in AF and VF data. Also, S data (black circles) show symmetric or asymmetric patterns that are well described by the model functions (black curves). Despite the visual quality of the fit, a goodness-of-fit test rejected the model for three observers (stars in Table 1) but these rejections seem spurious, as discussed next. Consider the case of observer 3 . The data vary smoothly up to intermediate positive auditory delays, and the model curves follow the path of the data very accurately. Yet, at the four longest positive auditory delays $S$ data (black circles) and VF data (blue circles) appear somewhat noisy, unlike AF data (red circles) at the same auditory delays. Thus, it seems that this observer was occasionally misreporting VF judgments as $S$ responses at long positive auditory delays. It is hard to imagine how an alternative model might produce curves that accommodate the smoothly varying data points on the left and center of the panel and then suddenly wind strangely to also accommodate the stray VF and S data points on the far right. It is even harder to agree to model rejection for observers 4 and 9 (for whom the $p$-values are also only marginally significant; see Table 1), since model curves follow the path of the data accurately across the panel except for occasional data points that deviate haphazardly from the path of the rest of the data. In all cases, the fitted model does not deviate systematically from the data for any observer but the stray location of some data points sometimes causes statistical rejection. An analysis of residuals identified the data points that caused rejection of the model for each observer, and these points are indicated by arrows in Figure 2. Note that, for observer 3, the only two misfitting data points involving AF and VF responses (indicated with red and blue arrows) imply very small observed frequencies and expected frequencies that are even smaller, a well-known cause of 

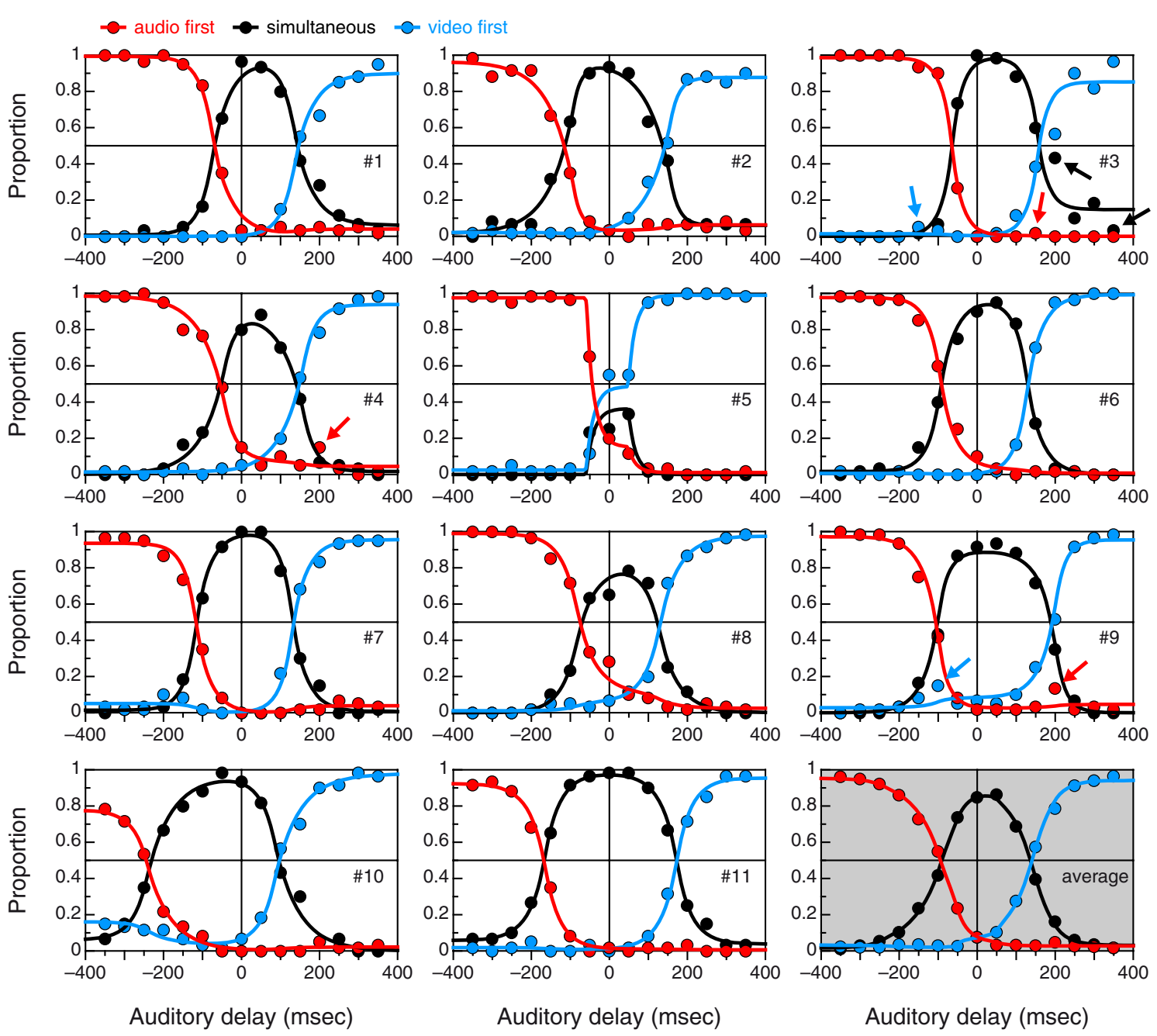

FIGURE 2 | Data and fitted curves in the flash-click experiment of van Eijk et al. (2008). The numeral in each panel denotes the observer; the panel at the bottom right shows summary results as averages of data and averages of the fitted functions across the 11 observers. Arrows in the panels for observers 3, 4, and 9 indicate the data points responsible for the misfit according to a residual analysis. improper model rejections (García-Pérez, 1994; García-Pérez and Núñez-Antón, 2001, 2004).

We do not report sample-wise goodness-of-fit analyses because there is no reason that the model should hold for a given sample as a whole (or just for $95 \%$ of the samples when the Type-I error rate is 0.05 ) but also because analyzing data aggregated across observers poses serious problems (Estes, 1956; Estes and Maddox, 2005). A subject-by-subject analysis of fit seems more reasonable and is the only means for identifying problematic assumptions in a model and potential replacements for them.

Although Figure 2 shows that the model fits the data adequately, we checked for parallelism of VF data (blue symbols) and inverted AF data (red symbols) for each observer, as follows. First, the locations $\Delta t_{\mathrm{VF}-\mathrm{PSS}}$ and $\Delta t_{\mathrm{AF}-\mathrm{PSS}}$ of the $50 \%$ point on $\Psi_{\mathrm{AF}}^{*}$ and $\Psi_{\mathrm{VF}}^{*}$ were determined. Then, we plotted $1-\Psi_{\mathrm{AF}}^{*}\left(\Delta t+\Delta t_{\mathrm{AF}-\mathrm{PSS}}\right)$ and $\Psi_{\mathrm{VF}}^{*}\left(\Delta t+\Delta t_{\mathrm{VF}-\mathrm{PSS}}\right)$, thus shifting them so that their $50 \%$ points coincide. Finally, we shifted the data analogously and also plotted them (after upside-down reversal of AF data). The results are shown in Figure 3 along with a summary panel for data and curves averaged across observers. The curves only show some deviations from parallelism for observers with relatively high error rates (observers 2, 3, 5, 7, and 10; see Table 1), and these deviations mostly affect the outer ends of each curve, where nonmonotonicity also occurs (see Figure 2). It is interesting to note that the data for which Allan (1975) and Ulrich (1987) reported a failure of parallelism also showed non-monotonic patterns at the outer ends, as is expected from intrusions from VF and AF judgments into AF and VF responses. This characteristic is more apparent in the plots that Jaskowski (1991) presented for the same data.

\section{A CASE OF OVERFITTING?}

Overfitting applies to models that have an unnecessarily large number of parameters and, thus, fit data by sheer volume of parameters. All the parameters in our model have empirical referents and, thus, their inclusion is justifiable. Each parameter produces a 
Table 1 | Estimated model parameters.

\begin{tabular}{|c|c|c|c|c|c|c|c|c|c|c|c|c|}
\hline Obs. & $G^{2}$ & $p$-value & $1 / \lambda_{a}$ & $1 / \lambda_{v}$ & $\delta$ & $\tau$ & $\varepsilon_{\mathrm{AF}}$ & $\varepsilon_{S}$ & $\varepsilon_{\mathrm{VF}}$ & $\mathrm{K}_{\mathrm{AF}-\mathrm{S}}$ & KS-AF & KVF-AF \\
\hline 2 & 19.39 & 0.514 & 59.92 & 20.96 & 125.28 & -33.28 & 0.035 & 0.030 & 0.123 & 0.407 & 1.000 & 0.510 \\
\hline 4 & $34.63 *$ & 0.041 & 57.90 & 29.69 & 101.74 & -59.02 & 0.014 & 0.078 & 0.062 & 0.000 & 0.990 & 0.731 \\
\hline 5 & 17.24 & 0.788 & 1.00 & 19.45 & 53.63 & 5.39 & 0.024 & 0.636 & 0.011 & 0.000 & 0.237 & 1.000 \\
\hline 6 & 22.60 & 0.137 & 29.64 & 36.90 & 111.47 & -15.34 & 0.022 & 0.029 & 0.007 & 0.740 & 1.000 & 1.000 \\
\hline 8 & 21.03 & 0.332 & 38.15 & 46.27 & 106.97 & -21.82 & 0.011 & 0.170 & 0.024 & 0.000 & 0.633 & 1.000 \\
\hline 9 & $34.11 *$ & 0.044 & 37.71 & 24.30 & 150.13 & -51.67 & 0.028 & 0.108 & 0.046 & 0.000 & 0.240 & 1.000 \\
\hline 10 & 25.02 & 0.561 & 35.85 & 61.25 & 163.60 & 79.60 & 0.222 & 0.032 & 0.022 & 0.274 & 0.000 & 1.000 \\
\hline 11 & 26.11 & 0.142 & 39.40 & 36.53 & 168.27 & -4.78 & 0.076 & 0.017 & 0.045 & 0.747 & 0.585 & 0.149 \\
\hline
\end{tabular}

The columns labeled $G^{2}$ and p-value respectively give the value and bootstrap significance level of the likelihood-ratio statistic; stars indicate rejection at the $5 \%$ significance level.
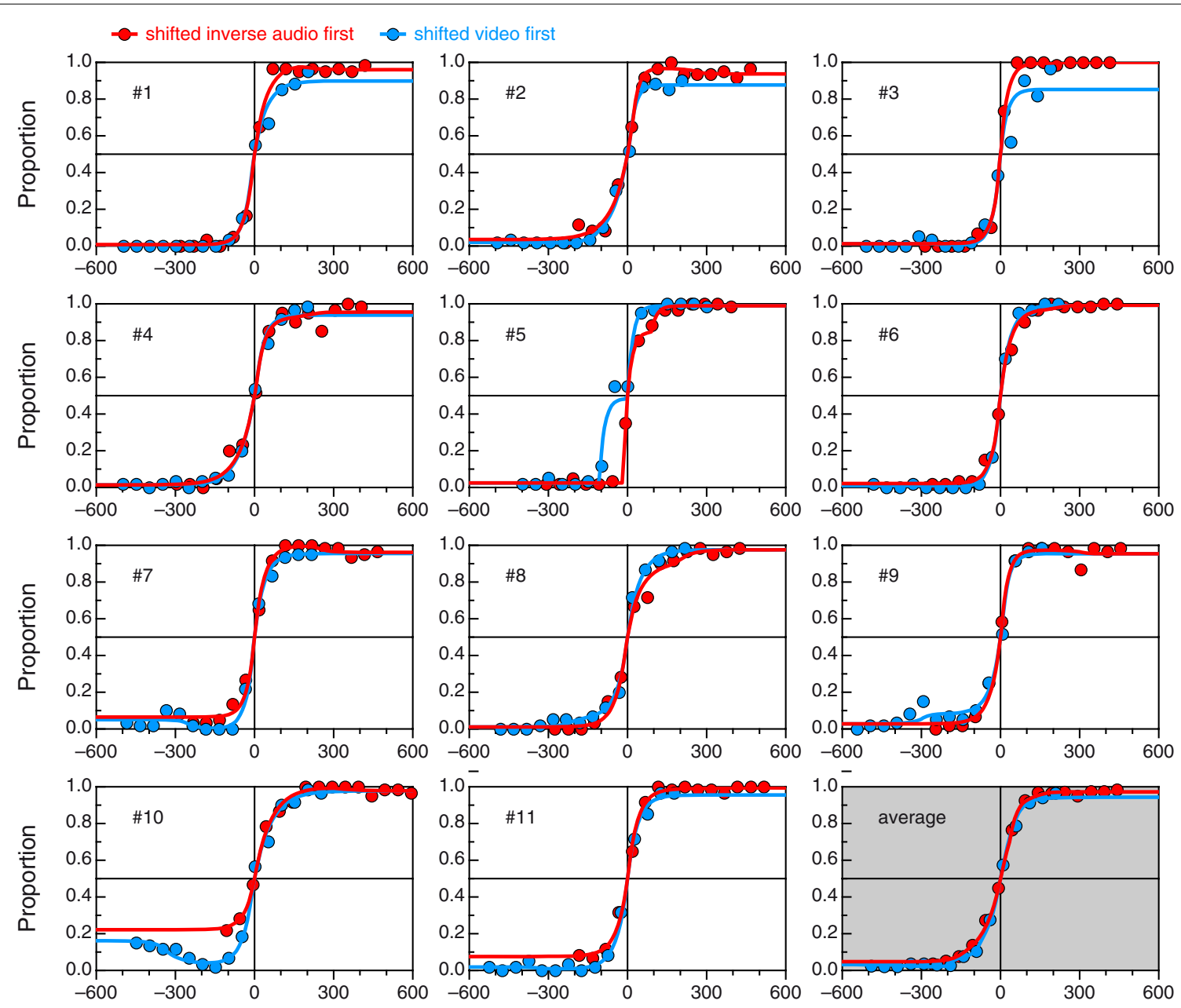

Time relative to the $50 \%$ point on each curve $(\mathrm{msec})$

FIGURE 3 | Test of parallelism. In comparison to Figure 2, AF and VF data and curves are merely shifted horizontally (and inverted upside-down in the case of AF data). 
distinctive and identifiable effect on the shape of the psychometric function, and these effects are not confounded (when lapses and the ensuing guesses are eliminated by design). And, more important, the data to which the model was fitted here show clear signs of these effects, which produce the non-monotonicity and lack of parallelism that conventional models cannot account for.

When considering the risk of overfitting, a comparison with the conventional approach that separately fits arbitrary functions to AF, S, and VF data is enlightening. In particular, on analyzing this same data set, van Eijk et al. (2008) fitted a four-parameter function to AF data, an independent four-parameter function to VF data, and still two other independent three-parameter functions to $S$ data (one of them for the ascending part and the other for the descending part). This yields a total of 14 parameters to describe the same data. Without the simplifications used by van Eijk et al., the number of free parameters under this approach may reach 16 . And not only the number of parameters is larger than that implied in our model, the functions fitted in this way cannot produce non-monotonic shapes for $\mathrm{AF}$ or VF data and the estimated parameters are uninterpretable in terms of underlying processes: they only indicate the slope, location, and asymptotes of the fitted functions.

Nevertheless, there is still the issue of whether some of the parameters of our model could be disposed of (particularly some of those representing response errors), or whether the simpler model with lapses would suffice to account for the data without response errors. The latter issue can be easily dispatched, as we showed above that a model including only lapses cannot produce nonmonotonic psychometric functions for AF and VF data. Since the data actually show these characteristics, the model with only lapses (Eqs 7a-7c) is disproved. To show that overfitting does not affect the model including response errors, we fitted simpler versions of it to the data, as described next.

In the simplest version, response errors are assumed to not occur at all, which implies making $\varepsilon_{\mathrm{AF}}=\varepsilon_{\mathrm{S}}=\varepsilon_{\mathrm{VF}}=0$ (wiping out $\kappa_{\mathrm{S}-\mathrm{AF}}, \kappa_{\mathrm{VF}-\mathrm{AF}}$, and $\kappa_{\mathrm{AF}-\mathrm{S}}$ along the way; see Eqs $\left.5 \mathrm{a}-5 \mathrm{c}\right)$ and leaving a model with only four free parameters $\left(\lambda_{\mathrm{a}}, \lambda_{\mathrm{v}}, \tau\right.$, and $\left.\delta\right)$. The results are shown in Figure 4, which reveals that forcing model
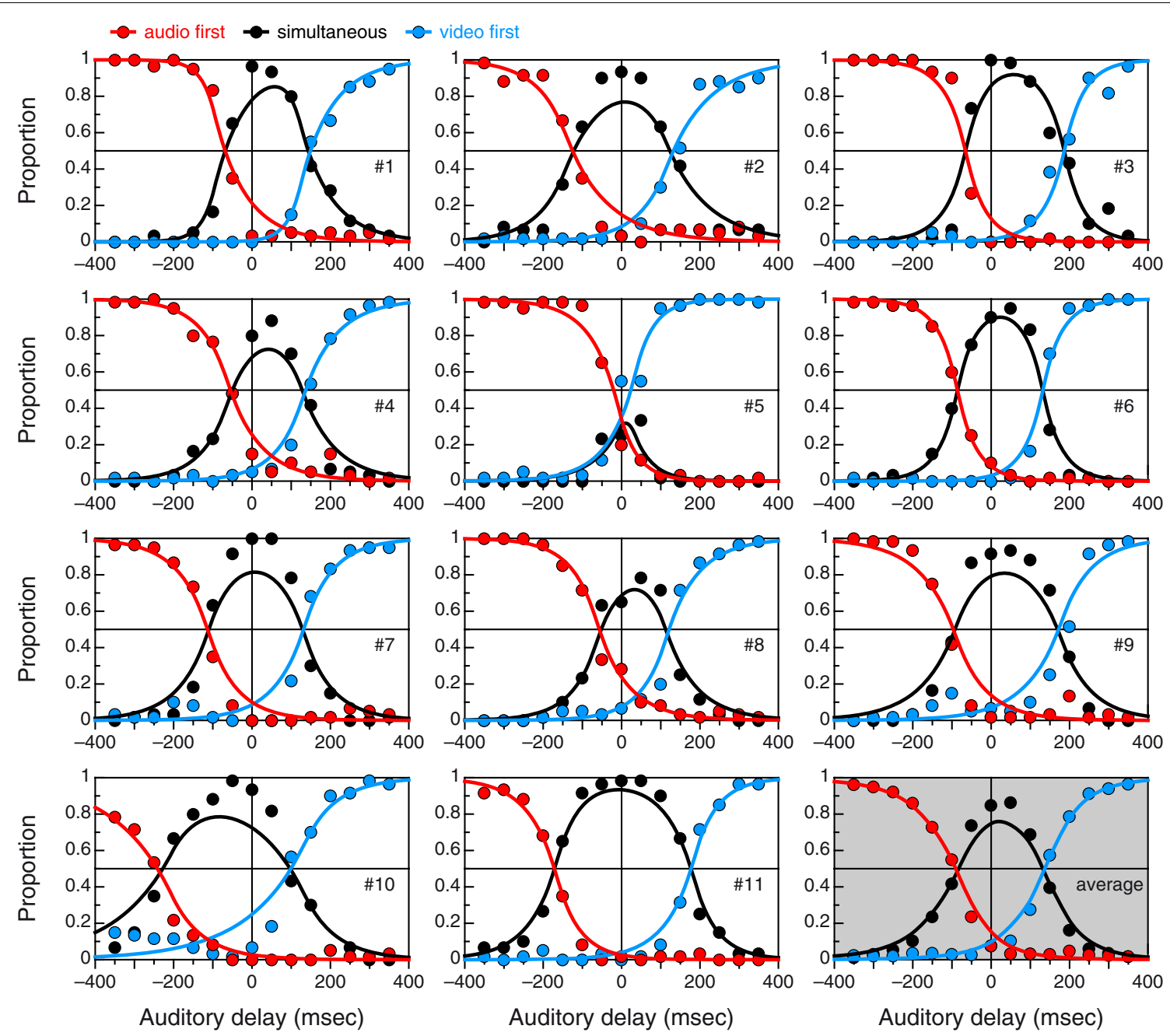

FIGURE 4 | Results of fitting a simpler version of the model in which no response errors are assumed to occur. Layout and graphical conventions as in Figure 2 
curves to have their asymptotes at 0 or 1 (as applicable) prevent them from accommodating the data, and the fit is particularly bad for observers whose data show clear signs of non-monotonicity or lack of parallelism. The $G^{2}$ statistic rejected the model for all observers (the largest $p$-value across observers was 0.002 ), a result that raises no concerns of potentially improper rejections given the obvious mismatch between the path described by the data and the path described by the curves in Figure 4.

In an intermediate version, all response errors were assumed to occur with the same probability (which implies making $\varepsilon_{\mathrm{AF}}=\varepsilon_{\mathrm{S}}=\varepsilon_{\mathrm{VF}}=\varepsilon$ ) and errors were further assumed to take all possible forms with the same probability (which implies making $\left.\kappa_{\mathrm{S}-\mathrm{AF}}=\kappa_{\mathrm{VF}-\mathrm{AF}}=\kappa_{\mathrm{AF}-\mathrm{S}}=1 / 2\right)$. This renders a model with five parameters $\left(\lambda_{\mathrm{a}}, \lambda_{\mathrm{v}}, \tau, \delta\right.$, and $\left.\varepsilon\right)$ for which Figure 5 shows the results. Again, the assumption that all response errors are equally likely prevents the model from fitting data that show clear signs to the contrary: this assumption forces the lower asymptote of all curves to be at the same height, and the upper asymptotes of curves for $\mathrm{AF}$ and VF data to also be at the same height, but the data say otherwise. This model was rejected for all observers except 2, 6, and 11, but it does not seem to do justice to the data for observer 2 (see data and curves in the bottom part of the panel for this observer in Figure 5).

We also tried out other simplifications, with analogous outcomes. Although some models fitted the data for some observers, accounting for the diversity of patterns of non-monotonicity and lack of parallelism across observers was impossible without allowance for all parameters. But this allowance does not imply that all parameters were actually useful to fit the data for all observers. Indeed, fitting the full model eliminates unnecessary error parameters by estimating them at or near zero. Consider the data for observer 3 in Figure $\mathbf{2}$ and note in Table $\mathbf{1}$ that the estimated values of $\varepsilon_{\mathrm{AF}}$ and $\varepsilon_{S}$ are nearly zero, which makes the estimated values of parameters $\kappa_{\mathrm{S}-\mathrm{AF}}$ and $\kappa_{\mathrm{AF}-\mathrm{S}}$ meaningless and immaterial. At the same time, the estimated value of $\varepsilon_{\mathrm{VF}}$ is 0.147 with an estimated $\kappa_{V F-A F}$ of 0 (or, equivalently, a derived value for $\kappa_{V F-S}$ of 1 , which are both meaningful because their companion error parameter $\varepsilon_{\mathrm{VF}}$ is not null). This matches what the data
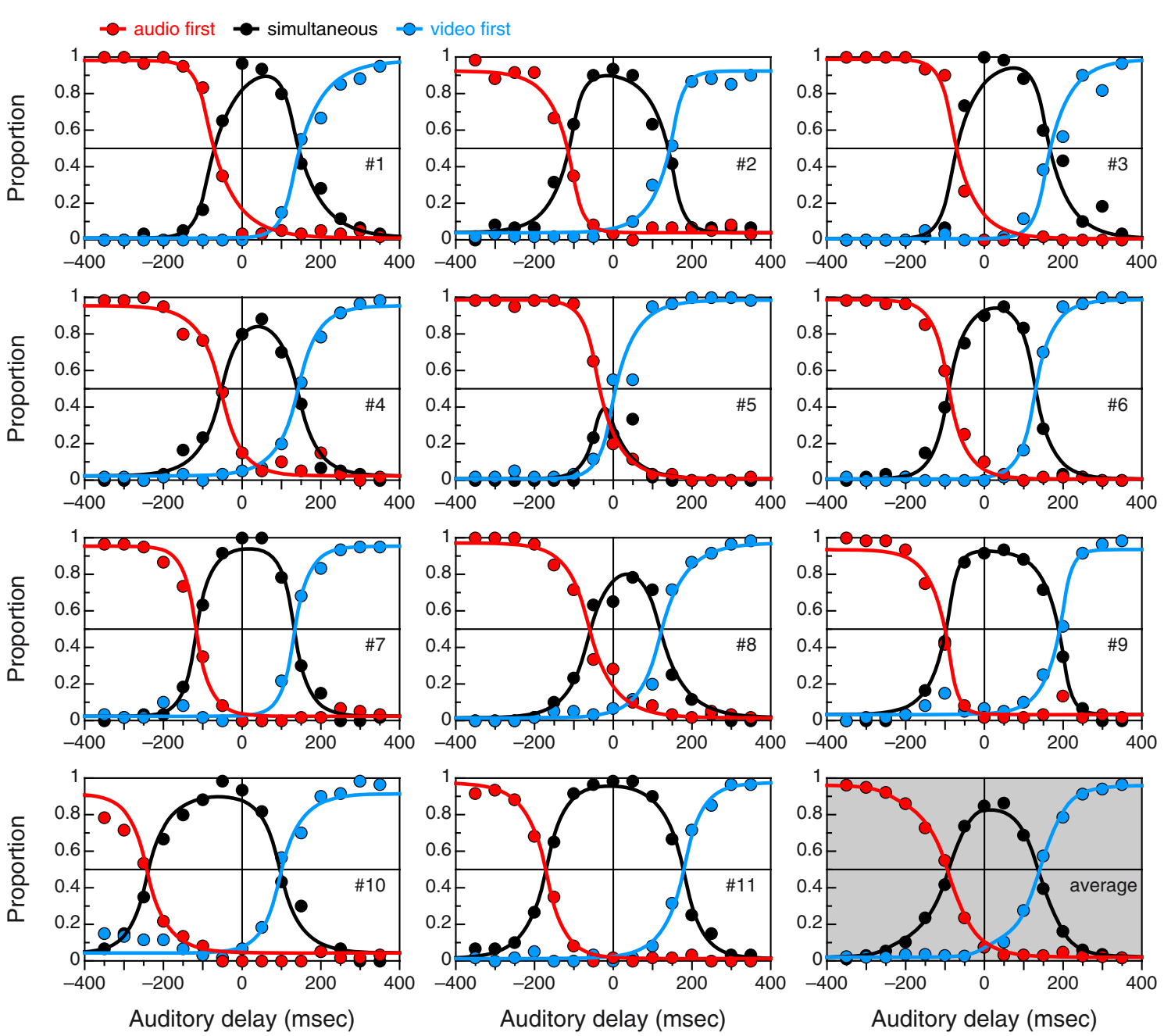

FIGURE 5 | Results of fitting a version of the model in which all types of response error are assumed to occur with the same probability and where the two types of misreported responses in case of error are assumed to occur with the same probability. Layout and graphical conventions as in Figure 2. 
for observer 3 suggest: only VF judgments seem to be occasionally misreported and always in the form of $\mathrm{S}$ responses. A similar analysis for observer 10 reveals the same match between features of the data and interpretation of parameter estimates, with the implicit elimination of unnecessary error parameters and their companion к's.

\section{PARAMETER IDENTIFICATION}

Four of the ten free parameters in the model $\left(\lambda_{\mathrm{a}}, \lambda_{\mathrm{V}}, \tau\right.$, and $\delta$ ) govern judgments and six $\left(\varepsilon_{\mathrm{AF}}, \varepsilon_{S}, \varepsilon_{\mathrm{VF}}, \kappa_{\mathrm{S}-\mathrm{AF}}, \kappa_{\mathrm{VF}-\mathrm{AF}}\right.$, and $\left.\kappa_{\mathrm{AF}-\mathrm{S}}\right)$ pertain to response errors. This may raise concerns of theoretical identifiability, that is, whether different sets of parameter values may produce the same psychometric functions $\Psi_{\mathrm{AF}}^{*}, \Psi_{\mathrm{S}}^{*}$, and $\Psi_{\mathrm{VF}}^{*}$. Since the problem cannot be addressed analytically, we took the approach of determining the extent to which the generating parameters were recovered from data sets essentially implying an infinite number of observations (because sampling error is not of concern here). One-thousand data sets were produced from random combinations of parameters with uniform distributions on $[1 / 80,1 / 20]$ for $\lambda_{\mathrm{a}}$ and $\lambda_{\mathrm{V}}$ (independently), on $[-80,80]$ for $\tau$, on $[20,150]$ for $\delta$, on $[0,0.1]$ for $\varepsilon_{\mathrm{AF}}, \varepsilon_{S}$, and $\varepsilon_{\mathrm{VF}}$ (independently), and on $[0,1]$ for $\kappa_{\mathrm{AF}-\mathrm{S}}, \kappa_{\mathrm{S}-\mathrm{AF}}$, and $\kappa_{\mathrm{VF}-\mathrm{AF}}$ (independently). The particular parameters that would produce each data set were inserted into Eqs $5 \mathrm{a}-5 \mathrm{c}$ and the resultant probabilities at auditory delays ranging from -350 to $350 \mathrm{~ms}$ in steps of $50 \mathrm{~ms}$ were transformed into (rounded off) expected numbers of AF, S, and VF responses across 10,000 putative trials per auditory delay. The model was subsequently fitted to each data set and parameter estimates were compared with the generating parameters in search for evidence of non-identifiability. The results are shown in Figure 6
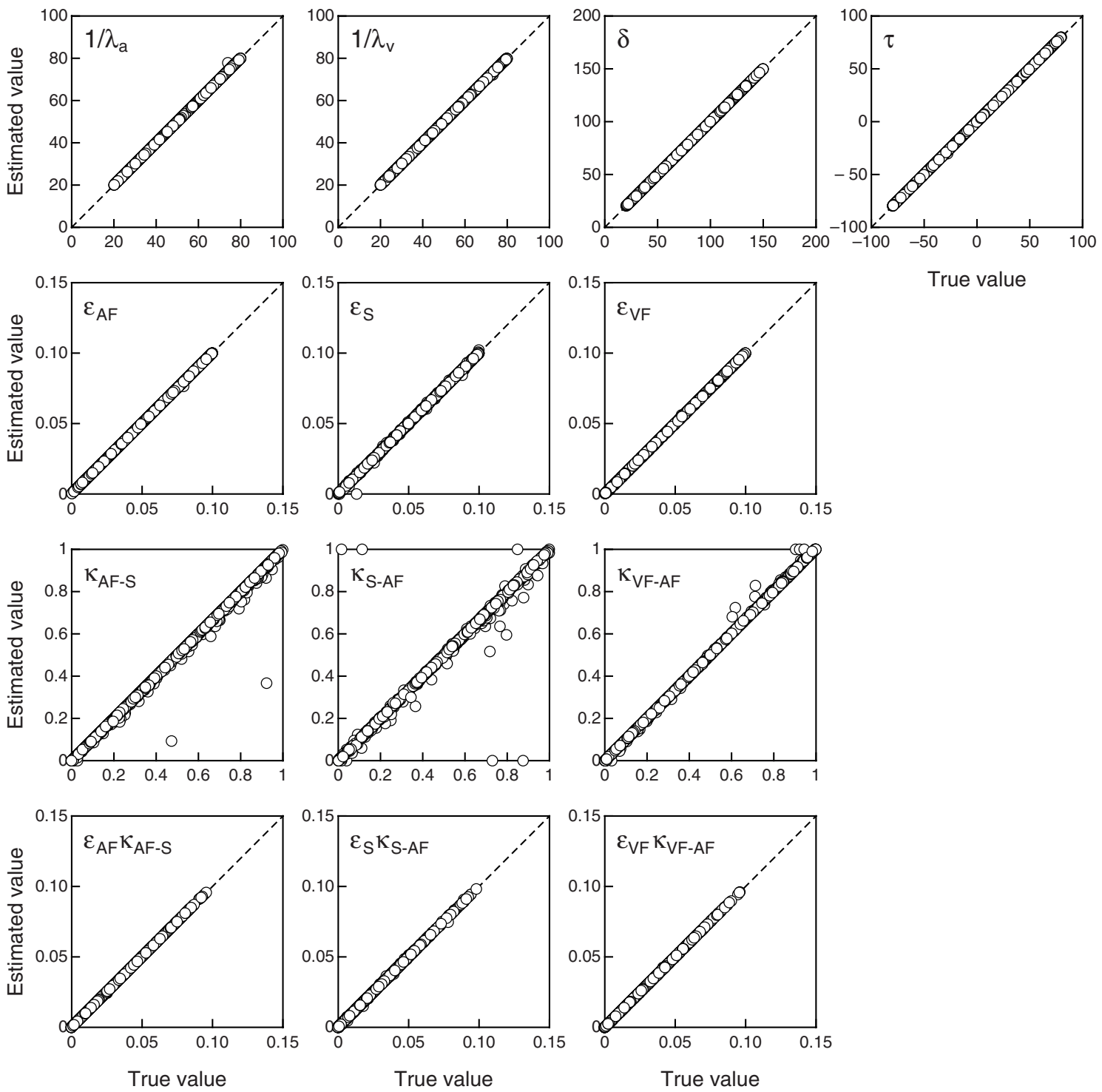

True value

True value

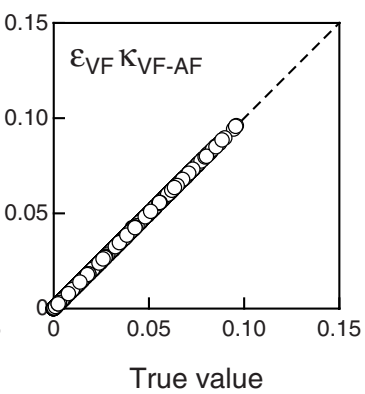

FIGURE 6 |Theoretical parameter identification. Each panel shows a scatter plot of estimated value against true value for the parameter indicated at the top-left corner, including the ten parameters of the model (first three rows) and the derived intrusion parameters (bottom row). Non-identifiability would show as data points falling along lines or curves other than the diagonal identity line in two or more panels within the first three rows. 
in the form of scatter plots of estimated against true parameter values. As is clear, all parameters are recovered with no evidence of non-identifiability. The minor misestimation problems affecting conditional probabilities $\kappa_{\mathrm{AF}-\mathrm{S}}, \kappa_{\mathrm{S}-\mathrm{AF}}$, and $\kappa_{\mathrm{VF}-\mathrm{AF}}$ (third row in Figure 4) are only a result of the fact that evidence on their actual values is limited or null when $\varepsilon_{\mathrm{AF}}, \varepsilon_{\mathrm{S}}$, and $\varepsilon_{\mathrm{VF}}$ are very small. The bottom row of Figure 6 shows that this misestimation is not a problem when intrusion parameters are considered.

A related issue is that of practical identifiability, that is, the extent to which model parameters can be recovered accurately from a finite number of observations affected by sampling error. When theoretical identifiability holds, as just documented, lack of practical identifiability speaks more of the scarcity of data than it speaks of the model itself. We looked into this issue under the same conditions discussed in the preceding paragraph (i.e., $N=15$ auditory delays and the same ranges of generating parameters), but now using 1000 sets of simulated counts (instead of expected counts) for numbers of trials ranging from 50 to 400 per auditory delay. The accuracy with which parameters were identified was measured through the concordance coefficient (Lin, 1989). The results are shown in Figure 7, which indicates how the concordance coefficient varies with number of trials per auditory delay. The parameters describing perception of temporal order $\left(\lambda_{\mathrm{a}}, \lambda_{\mathrm{v}}\right.$, $\tau$, and $\delta$; first panel), the error parameters $\varepsilon_{\mathrm{AF}}$ and $\varepsilon_{\mathrm{VF}}$ (second panel), and the conditional probabilities $\kappa_{\mathrm{AF}-\mathrm{S}}$ and $\kappa_{\mathrm{VF}-\mathrm{AF}}$ (third panel) can be sufficiently accurately recovered with 100 trials per auditory delay. Yet, $\varepsilon_{S}$ and $\kappa_{S-A F}$ are harder to recover, a result of the fact that misreports of S judgments (which would occur at short auditory delays) are harder to identify than misreports of AF or VF judgments (which would occur at long positive and negative auditory delays) ${ }^{1}$.

In sum, model parameters are theoretically and practically identifiable with only a slight misestimation of error parameters affecting $\mathrm{S}$ judgments, something that does not hamper the

${ }^{1}$ For some mixture models, it has been reported that the EM algorithm yields more favorable estimation performance in terms of bias and variability of estimates (e.g., Lawrence, 2010). Checking out whether (and to what extent) the EM algorithm outperforms the quasi-Newton method used here is an area of future research. estimation of the relevant parameters governing perception of temporal order $\left(\lambda_{\mathrm{a}}, \lambda_{\mathrm{v}}, \tau\right.$, and $\left.\delta\right)$. In any case, error rates are nuisance parameters that are known to be generally difficult to estimate accurately (García-Pérez and Alcalá-Quintana, 2005) but it is also known that including them in the functions to be fitted increases the precision with which relevant parameters can be estimated (see also García-Pérez and Alcalá-Quintana, 2010a,b, 2011). Actually, allowance for error or lapse parameters in model psychometric functions for binary tasks is routine practice in visual psychophysics (Kingdom and Prins, 2010), but not so much in psychoacoustics or in research on prior entry or perception of temporal order. More thorough studies of parameter recovery also involving alternative estimation methods should be carried out, but these lie beyond the scope of this paper.

\section{CONCLUSION}

SJ3 data can be described by independent-channels models when response errors are considered, as lack of monotonicity and parallelism results from these errors. Lapses of attention, in turn, produce lack of parallelism but not lack of monotonicity. Response errors are often made inadvertently and, then, cannot be easily removed from the data. Yet, the contaminating influence of lapses can be easily removed by allowing observers to abort trials in which they missed the stimuli, instead of forcing them to indicate a judgment that they could not possibly have made. Implementing this feature in the response interface is highly recommendable.

Reinstating independent-channels models with their interpretable parameters will contribute to a more precise analysis of the effects of experimental manipulations in studies of prior entry or temporal recalibration. Recourse to these models will also be useful in general studies on the perception of temporal order under different stimulus conditions (see van Eijk et al., $2008,2010)$ and for the analysis of observed differences in performance across tasks (i.e., the ternary SJ3 task considered here, its binary version SJ2 in which observers only indicate whether presentation was simultaneous or sequential, and the temporal order judgment task TOJ in which observers are forced to respond AF or VF without allowance for an S response; see García-Pérez and Alcalá-Quintana, submitted).

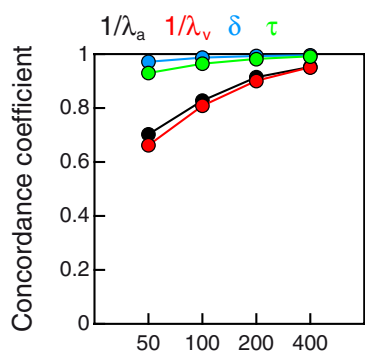

No. trials per level

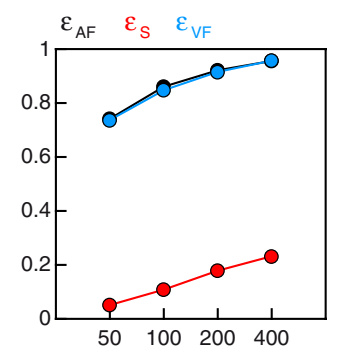

No. trials per level

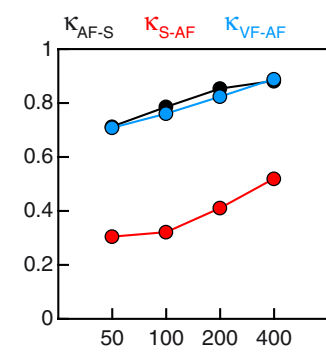

No. trials per level

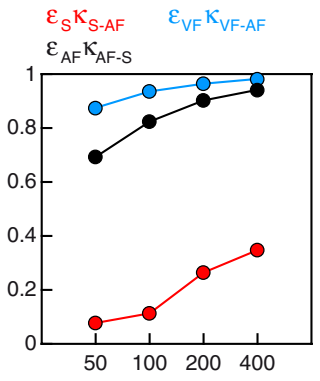

No. trials per level
FIGURE 7 | Practical parameter identification. Each panel shows how recovery of the 10 parameters of the model (first three panels) and the derived intrusion parameters (fourth panel) varies with the number of observations collected at each of 15 auditory delays ranging from -350 to $350 \mathrm{~ms}$ in steps of $50 \mathrm{~ms}$. An asymptotic 0.05-size chi-square test yielded empirical rejection rates of 4.3, 4.1, 5.4 , and $5.5 \%$ as the number of observations per auditory delay increased from 50 to 400; an analogous asymptotic 0.05-size likelihood-ratio test yielded inaccurate empirical rejection rates of 8.1, 8.0, 7.3, and $8.3 \%$. 
The model presented here emphasizes the distinction between unobservable judgments and observed responses, where the latter are not direct indicators of the former when response errors occur. Evidence of response errors is conspicuous in empirical data and errors are likely to occur more often when reaction times are also measured, due to the pressure to respond quickly and the ternary nature of the task (i.e., observers have to be quick but without mistaking which of the three response keys they have to press to indicate their judgment). Because response errors are not unlikely in these cases, fitting a model that allows for them is thus of outmost importance in these cases. User-friendly software packages (in матLAв and R) are under development for fitting the model

\section{REFERENCES}

Allan, L. G. (1975). The relationship between judgments of successiveness and judgments of order. Percept. Psychophys. 18, 29-36.

Collett, D. (2003). Modelling Binary Data, 2nd Edn. Boca Raton: CRC Press.

Colonius, H., and Diederich, A. (2011). Computing an optimal time window of audiovisual integration in focused attention tasks: illustrated by studies on effect of age and prior knowledge. Exp. Brain Res. 212, 327-337.

Diederich, A., and Colonius, H. (2011). "Modeling multisensory processes in saccadic responses: time-windowof-integration model," in The Neural Bases of Multisensory Processes, eds M. M. Murray and M. T. Wallace (Boca Raton: CRC Press), 253-276.

Estes, W. K. (1956). The problem of inference from curves based on group data. Psychol. Bull. 53, 134-140.

Estes, W. K., and Maddox, W. T. (2005). Risks of drawing inferences about cognitive processes from model fits to individual versus average performance. Psychon. Bull. Rev. 12, 403-408.

Fujisaki, W., and Nishida, S. (2009). Audio-tactile superiority over visuo-tactile and audio-visual combinations in the temporal resolution of synchrony perception. Exp. Brain Res. 198, 245-259.

García-Pérez, M. A. (1994). Parameter estimation and goodness-offit testing in multinomial models. Br. J. Math. Stat. Psychol. 47, 247-282.

García-Pérez, M. A., and AlcaláQuintana, R. (2005). Sampling plans for fitting the psychometric function. Span. J. Psychol. 8, 256-289.

García-Pérez, M. A., and AlcaláQuintana, R. (2010a). The difference model with guessing explains interval bias in two-alternative forcedchoice detection procedures. J. Sens. Stud. 25, 876-898.

García-Pérez, M. A., and AlcaláQuintana, R. (2010b). Reminder and 2AFC tasks provide similar estimates of the difference limen: a reanalysis of data from Lapid, Ulrich, and Rammsayer (2008) and a discussion of Ulrich and Vorberg (2009). Atten. Percept. Psychophys. 72, 1155-1178. [A correction has been published: Atten. Percept. Psychophys. 2012, 74, 489-492.]

García-Pérez, M. A., and AlcaláQuintana, R. (2011). Interval bias in 2AFC detection tasks: sorting out the artifacts. Atten. Percept. Psychophys. 73, 2332-2352.

García-Pérez, M. A., and NúñezAntón, V. (2001). Small-sample comparisons for power-divergence goodness-of-fit statistics for symmetric and skewed simple null hypotheses. J. Appl. Stat. 28, 855-874.

García-Pérez, M. A., and Núñez-Antón, V. (2004). Small-sample comparisons for goodness-of-fit statistics in one-way multinomials with composite hypotheses. J. Appl. Stat. 31, 161-181.

Harrar, V., and Harris, L. R. (2008). The effect of exposure to asynchronous audio, visual, and tactile stimulus combinations on the perception of simultaneity. Exp. Brain Res. 186, 517-524.

Heath, R. A. (1984). Response time and temporal order judgement in vision. Aust. J. Psychol. 36, 21-34.

Jaskowski, P. (1991). Two-stage model for order discrimination. Percept. Psychophys. 50, 76-82.

Jaskowski, P. (1993). Selective attention and temporal-order judgment. Perception 22, 681-689.

Kingdom, F. A. A., and Prins, N. (2010). Psychophysics: A Practical Introduction. London: Academic Press.

presented here to SJ3 data (Alcalá-Quintana and García-Pérez, submitted).

\section{ACKNOWLEDGMENTS}

This research was supported by grant PSI2009-08800 from Ministerio de Ciencia e Innovación (Spain). We thank Rob van Eijk for kindly supplying their data. We also thank Michael Lawrence and Mark Yates for their comments on an earlier draft. Correspondence concerning this article should be sent to Miguel A. García-Pérez, Departamento de Metodología, Facultad de Psicología, Universidad Complutense, Campus de Somosaguas, 28223 Madrid, Spain (e-mail: miguel@psi.ucm.es).

Kristofferson, A. B., and Allan, L. G. (1973). "Successiveness and duration discrimination," in Attention and Performance IV, ed. S. Kornblum (New York: Academic Press), 737-749.

Lawrence, M. A. (2010). Estimating the probability and fidelity of memory. Behav. Res. Methods 42, 957-968.

Lin, L. I.-K. (1989). A concordance correlation coefficient to evaluate reproducibility. Biometrics 45, 255-268.

Nicholls, M. E. R., Lew, M., Loetscher, T., and Yates, M. J. (2011). The importance of response type to the relationship between temporal order and numerical magnitude. Atten. Percept. Psychophys. 73, 1604 1613.

Numerical Algorithms Group. (1999). NAG Fortran Library Manual, Mark 19. Oxford: Numerical Algorithms Group.

Occelli, V., Spence, C., and Zampini, M. (2011). Audiotactile interactions in temporal perception. Psychon. Bull. Rev. 18, 429-454.

Schneider, K. A., and Bavelier, D. (2003). Components of visual prior entry. Cogn. Psychol. 47, 333-366.

Shore, D. I., Spry, E., and Spence, C. (2002). Confusing the mind by crossing the hands. Cogn. Brain Res. 14, 153-163.

Spence, C., Baddeley, R., Zampini, M., James, R., and Shore, D. I. (2003). Multisensory temporal order judgments: when two locations are better than one. Percept. Psychophys. 65, 318-328.

Spence, C., and Parise, C. (2010). Priorentry: a review. Conscious. Cogn. 19, 364-379.

Sternberg, S., and Knoll, R. L. (1973). "The perception of temporal order: fundamental issues and a general model," in Attention and Performance $I V$, ed. S. Kornblum (New
York: Academic Press), 629-685.

Stetson, C., Cui, X., Montague, P. R., and Eagleman, D. M. (2006). Motorsensory recalibration leads to an illusory reversal of action and sensation. Neuron 51, 651-659.

Stone, J. V., Hunkin, N. M., Porrill, J., Wood, R., Keeler, V., Beanland, M. Port, M., and Porter, N. R. (2001). When is now? Perception of simultaneity. Proc. R. Soc. Lond. B Biol. Sci. 268, 31-38.

Swanson, W. H., and Birch, E. E. (1992). Extracting thresholds from noisy psychophysical data. Percept. Psychophys. 51, 409-422.

Ulrich, R. (1987). Threshold models of temporal-order judgments evaluated by a ternary response task. Percept. Psychophys. 42, 224-239.

van Eijk, R. L. J., Kohlrausch, A., Juola, J. F., and van de Par, S. (2008). Audiovisual synchrony and temporal order judgments: effects of experimental method and stimulus type. Percept. Psychophys. 70, 955-968.

van Eijk, R. L. J., Kohlrausch, A., Juola, J. F., and van de Par, S. (2010). Temporal order judgment criteria are affected by synchrony judgment sensitivity. Atten. Percept. Psychophys. 72, 2227-2235.

Vatakis, A., Navarra, J., Soto-Faraco, S., and Spence, C. (2008). Audiovisual temporal adaptation of speech: temporal order versus simultaneity judgments. Exp. Brain Res. 185, 521-529.

Vroomen, J., and Keetels, M. (2010). Perception of intersensory synchrony: a tutorial review. Atten. Percept. Psychophys. 72, 871-874.

Wichmann, F. A., and Hill, N. J. (2001). The psychometric function: I. Fitting, sampling, and goodness of fit. Percept. Psychophys. 63, 1293-1313.

Yates, M. J., and Nicholls, M. E. R. (2009). Somatosensory prior entry. Atten. Percept. Psychophys. 71, 847-859. 
Yates, M. J., and Nicholls, M. E. R. (2011). Somatosensory prior entry assessed with temporal order judgments and simultaneity judgments. Atten. Percept. Psychophys. 73, 1586-1603.

Conflict of Interest Statement: The authors declare that the research was conducted in the absence of any commercial or financial relationships that could be construed as a potential conflict of interest.

Received: 10 January 2012; accepted: 13 March 2012; published online: 04 April 2012.
Citation: García-Pérez MA and AlcaláQuintana $R$ (2012) Response errors explain the failure of independentchannels models of perception of temporal order. Front. Psychology 3:94. doi: 10.3389/fpsyg.2012.00094

This article was submitted to Frontiers in Perception Science, a specialty of Frontiers in Psychology.
Copyright (C) 2012 García-Pérez and Alcalá-Quintana. This is an openaccess article distributed under the terms of the Creative Commons Attribution Non Commercial License, which permits non-commercial use, distribution, and reproduction in other forums, provided the original authors and source are credited. 